\title{
A formula of Lascoux-Leclerc-Thibon and representations of symmetric groups
}

\author{
Hideaki Morita • Tatsuhiro Nakajima
}

Received: 23 August 2005 / Accepted: 5 December 2005

(C) Springer Science + Business Media, LLC 2006

\begin{abstract}
We consider Green polynomials at roots of unity, corresponding to partitions which we call $l$-partitions. We obtain a combinatorial formula for Green polynomials corresponding to $l$-partitions at primitive $l$ th roots of unity. The formula is rephrased in terms of representation theory of the symmetric group.
\end{abstract}

\section{Introduction}

Green polynomials [5] at roots of unity were first considered by A. Morris and N. Sultana. They study in [15] Hall-Littlewood symmetric functions at roots of unity in connection with modular representation theory of the symmetric group. They conjecture a certain recurrence formula for Green polynomials at roots of unity corresponding to rectangle partitions. The orders of the roots are restricted to the multiplicity of the rectangle partition. The conjecture was proved by A. Lascoux, B. Leclerc and J. -Y. Thibon [9], as an application of their result on Hall-Littlewood functions at roots of unity. They showed in [8] a factorization formula for Hall-Littlewood functions at roots of unity, and a plethystic formula for the case corresponding to rectangle partitions. These formulas play a key role in the proof of the conjecture.

In this paper, we consider Green polynomials at roots of unity, corresponding to partitions which we call $l$-partitions. The $l$-partitions are defined to be the partitions whose multiplicities are all divisible by a fixed positive integer $l$. Lascoux-LeclercThibon showed that Green polynomials corresponding to $l$-partitions at primitive $l$ th roots of unity are described by the inner product of complete symmetric functions

H. Morita $(\bowtie)$

School of Science, Tokai University, Hiratsuka 259-1292, Japan

e-mail: moritah@msg.boglobe.ne.jp

T. Nakajima

Faculty of Economics, Meikai University, Urayasu 279-8550, Japan

e-mail: tatsu@ka2.so-net.ne.jp 
and power-sum symmetric functions (see, e.g., [2]). We obtain in the present article an explicit formula for the inner product in terms of partitions, and hence obtain a combinatorial description of those Green polynomials at primitive $l$ th roots of unity.

We also consider the formula in terms of representation theory of the symmetric group. Let $n$ be a positive integer, and $S_{n}$ the symmetric group of $n$ letters. There corresponds to each partition $\mu$ of positive integer $n$ a graded $S_{n}$-module $R_{\mu}$, the DeConciniProcesi-Tanisaki algebra corresponding to $\mu$. The DeConcini-Procesi-Tanisaki algebra was first considered by DeConcini-Procesi [3] as an algebraic model for the Springer representation $[10,19]$ of Weyl groups. It is known that the Green polynomial corresponding to a partition $\mu$ gives the graded character value of the graded $S_{n}$-module $R_{\mu}$. We understand our combinatorial formula in terms of the representation theory of $S_{n}$ on these graded representations, in the case where the partition is an $l$-partition. Indeed, the formula is rephrased as a representation theoretical interpretation of a certain combinatorial property of the graded module $R_{\mu}$.

If $\mu$ is an $l$-partition, then the subspaces of $R_{\mu}$, defined by taking the direct sum of homogeneous components whose degrees are congruent modulo $l$, have the same dimension $\left(\operatorname{dim} R_{\mu}\right) / l$. The property is referred to in this paper as 'coincidence of dimension'. In this case, our formula states that these submodules of $R_{\mu}$ of equal dimension are induced from representations of certain subgroup of $S_{n}$, which are all onedimensional. In fact, this representation theoretical interpretation of the coincidence of dimensions of $R_{\mu}$ is equivalent to the following $S_{n} \times C_{l}$-module isomorphism:

$$
R_{\mu} \cong \operatorname{Ind}_{S_{\mu}}^{S_{n}} R_{\emptyset},
$$

where $S_{\mu}$ denotes the Young subgroup corresponding to the partition $\mu, R_{\emptyset}$ the DeCoicini-Procesi-Tanisaki algebra corresponding to the empty partition $\varnothing$ which is isomorphic to $\mathbf{C}$, viewed as the trivial representation of $S_{\mu}$. As $S_{n}$-modules, this isomorphism is well known (c.f., [4]). The point which should be respected here is that the isomorphism includes the action of the cyclic group $C_{l}$. It can be regarded that the isomorphism partially recovers the grading in Lusztig's induction theorem of the Springer representations for the symmetric groups (c.f., [17]).

The paper is organized as follows. In Section 2, we collect fundamental facts on Hall-Littlewood functions and Green polynomials at roots of unity. Formulas of Lascoux-Leclerc-Thibon on these materials are reviewed. In Section 3, we obtain a combinatorial formula for the Green polynomials corresponding to $l$-partitions at primitive $l$ th roots of unity. In Section 4 , we consider, as an application of the formula, the coincidence of dimensions of the algebra $R_{\mu}$ in terms of representation theory of $S_{n}$. In Section 5, we make a final remark.

\section{Green polynomials and modified Hall-Littlewood functions}

Let $x=\left(x_{1}, x_{2}, \ldots, x_{n}, \ldots\right)$ denote a set of infinite variables, and $\Lambda$ the ring of symmetric functions with the variables $x_{1}, x_{2}, \ldots, x_{n}, \ldots$ We follow [11] for notation on symmetric functions. Let $p_{\rho}(x)$ denote the power-sum symmetric function corresponding to a partition $\rho \vdash n$, and $P_{\mu}(x ; q)$ denote the Hall-Littlewood symmetric function corresponding to a partition $\mu \vdash n$. Since the Hall-Littlewood functions form 
a $\mathbf{Z}[q]$-basis of the ring of symmetric functions $\Lambda[q]=\Lambda \otimes \mathbf{Z}[q]$, we can expand $p_{\rho}(x)$ into a linear combination of $P_{\mu}(x ; q)$ 's with the coefficients in $\mathbf{Z}[q]$ :

$$
p_{\rho}(x)=\sum_{\mu \vdash n} X_{\rho}^{\mu}(q) P_{\mu}(x ; q), \quad X_{\rho}^{\mu}(q) \in \mathbf{Z}[q] .
$$

Then the Green polynomials $Q_{\rho}^{\mu}(q)$ are defined by

$$
Q_{\rho}^{\mu}(q)=q^{n(\mu)} X_{\rho}^{\mu}\left(q^{-1}\right)
$$

They are polynomials in $q$ with integer coefficients, and the degree is given by $n(\mu)=$ $\sum_{i=1}^{d}(i-1) \mu_{i}$, where $\mu=\left(\mu_{1}, \mu_{2}, \ldots, \mu_{d}\right)$.

The aim of the present article is to consider the Green polynomials at roots of unity for the partitions which we call the l-partitions, that is, the partitions $\mu=$ $\left(1^{m_{1}} 2^{m_{1}} \cdots n^{m_{n}}\right)$ for which the multiplicities $m_{1}, m_{2}, \ldots, m_{n}$ are all divisible by a positive integer $l$. We also use a symbol $m_{i}(\mu)$ to depict the multiplicity of $i$ in a partition $\mu$. We recall here a result of Lascoux, Leclerc and Thibon on the Green polynomials for $l$-partitions at $l$ th roots of unity (see, e.g., [2, Theorem 9.7]). Let $l$ be a positive integer, and $\mu=\left(1^{m_{1}} 2^{m_{2}} \cdots n^{m_{n}}\right)$ an $l$-partition. Then $\mu^{1 / l}$ denotes the partition $\left(1^{q_{1}} 2^{q_{2}} \cdots n^{q_{n}}\right)$, where $m_{i}=l q_{i}$ for each $i=1,2, \ldots n$. For a partition $v$ of a positive intger, let $l v$ denote the partition obtained by multiplying each component of $v$ by $l$. Let $h_{v}$ denotes the complete symmetric function corresponding to the partition $v$, and $\langle f, g\rangle$ denotes the usual inner product of the ring of symmetric functions, defined by

$$
\left\langle p_{\lambda}(x), p_{\mu}(x)\right\rangle=z_{\lambda} \delta_{\lambda \mu},
$$

where $z_{\lambda}=1^{k_{1}} k_{1} ! 2^{k_{2}} k_{2} ! \cdots n^{k_{n}} k_{n}$ ! for $\lambda=\left(1^{k_{1}} 2^{k_{2}} \cdots n^{k_{n}}\right)$, and $\delta_{\lambda \mu}$ the Kronecker delta.

Proposition 1 (Lascoux-Leclerc-Thibon). Let $l$ be a positive integer, and $\mu$ an $l$ partition. Let $\zeta_{l}$ be a primitive lth root of unity. Then it holds that

$$
X_{\rho}^{\mu}\left(\zeta_{l}\right) \neq 0 \Longrightarrow \rho=l v
$$

for some partition $v \vdash n / l$. In this case, we have

$$
X_{\rho}^{\mu}\left(\zeta_{l}\right)=(-1)^{(l-1)\left|\mu^{1 / l}\right|} l^{l(\nu)}\left\langle p_{\nu}, h_{\mu^{1 / l}}\right\rangle
$$

The following two results on Hall-Littlewood functions at roots of unity, due to Lascoux-Leclerc-Thibon, play a key role in the proof of Proposition 1, of which we also make use in the present article. Let $\mu$ be a partition of $n$. Let $Q_{\mu}(x ; q)$ denote the symmetric function with parameter $q$, defined by

$$
Q_{\mu}(x ; q):=b_{\mu} P_{\mu}(x ; q),
$$


where $b_{\mu}=\prod_{i=1}^{n}(1-q)\left(1-q^{2}\right) \cdots\left(1-q^{m_{i}(\mu)}\right)$. This symmetric function $Q_{\mu}(x ; q)$ is also called the Hall-Littlewood symmetric function. These two classes $\left\{P_{\lambda}(x ; q)\right\}$, $\left\{Q_{\lambda}(x ; q)\right\}$ of symmetric functions are dual to each other with respect to the HallLittlewood inner product [11, p. 225]. Let $Q_{\mu}^{\prime}(x ; q)$ denote the modified HallLittlewood symmetric function, which is defined by

$$
Q_{\mu}^{\prime}(x ; q)=Q_{\mu}\left(\frac{x}{1-q} ; q\right),
$$

i.e., the symmetric function obtained by replacing the variable $x=\left(x_{1}, x_{2}, \ldots\right)$ with $x / 1-q=\left(x_{1}, q x_{1}, q^{2} x_{1}, \ldots ; x_{2}, q x_{2}, q^{2} x_{2}, \ldots\right)$ in $Q_{\mu}(x ; q)$. Then it is not difficult to see that the Hall-Littlewood symmetric functions $\left\{P_{\lambda}(x ; q)\right\}$ and modified HallLittlewood functions $\left\{Q_{\lambda}^{\prime}(x ; q)\right\}$ are dual to each other with respect to the usual inner product of the ring of symmetric function $\Lambda[q]$. It immediately follows from this fact that the Green polynomial $X_{\rho}^{\mu}(q)$ is obtained by

$$
X_{\rho}^{\mu}(q)=\left\langle Q_{\mu}^{\prime}(x ; q), p_{\rho}\right\rangle
$$

for all partitions $\mu, \rho \vdash n$.

Proposition 2 ([9, Theorem 2.1]). Let $\mu$ be a partition $\mu=\left(1^{m_{1}} 2^{m_{2}} \cdots n^{m_{n}}\right)$ of $n$. Let $l$ be a positive integer. Suppose that $m_{i}=l q_{i}+r_{i}, 0 \leq r_{i} \leq l-1$ for each $i=$ $1,2, \ldots, n$. Then it holds that

$$
Q_{\mu}^{\prime}\left(x ; \zeta_{l}\right)=Q_{\bar{\mu}}^{\prime}\left(x ; \zeta_{l}\right) \prod_{i=1}^{n}\left\{Q_{\left(^{l}\right)}^{\prime}\left(x ; \zeta_{l}\right)\right\}^{q_{i}},
$$

where $\bar{\mu}$ denotes the partition $\left(1^{r_{1}} 2^{r_{2}} \cdots n^{r_{n}}\right)$.

Proposition 3 ([9, Theorem 2.2]). Let l and $r$ be a positive integers. Then we have

$$
Q_{\left(r^{l}\right)}^{\prime}\left(x ; \zeta_{l}\right)=(-1)^{(l-1) r}\left(p_{l} \circ h_{r}\right)(x)
$$

where $\left(p_{l} \circ h_{r}\right)(x)$ denotes the plethysm of the complete symmetric function $h_{r}$ by the power-sum symmetric function $p_{l}$.

\section{Explicit formula}

Let $l>1$ be a positive integer, and $\mu \vdash n$ an $l$-partition. Let $q_{i}=m_{i} / l$ for each $i=1,2, \ldots, n$. Recall that $\mu^{1 / l}$ is by definition the partition $\left(1^{q_{1}} 2^{q_{2}} \cdots n^{q_{n}}\right)$ of $n / l$. (In fact, $q_{i}=0$ for all $i>n / l$.) Let $v=\left(v_{1}, v_{2}, \ldots, v_{r}\right)$ be a partition, and $\underline{\kappa}=\left(\kappa^{(1)}, \kappa^{(2)}, \ldots, \kappa^{(r)}\right)$ a sequence of partitions. Then $\underline{\kappa} \vdash v$ means that $\kappa^{(i)} \vdash v_{i}$ for each $i=1,2, \ldots, r$, and $\underline{\kappa}$ is called a partition of $v$. If $\underline{\kappa}$ is a partition of $v$, then $l_{\underline{\kappa}}$ denotes the partition of a positive integer whose components are those of $\underline{\kappa}$ multiplied 铂 Springer 
by $l$. For a partition $\underline{\kappa}=\left(\kappa^{(i)}\right) \vdash v$, define

$$
m_{k}(\underline{\kappa}):=\sum_{i \geq 1} m_{k}\left(\kappa^{(i)}\right)
$$

for each $k$, and

$$
m_{\underline{\kappa}}:=\prod_{k \geq 1}\left(\begin{array}{c}
m_{k}(\underline{\kappa}) \\
m_{k}\left(\kappa^{(1)}\right), m_{k}\left(\kappa^{(2)}\right), \ldots
\end{array}\right),
$$

where $\left(\begin{array}{c}i \\ j, k, \ldots\end{array}\right)$ denotes the multinomial coefficient.

Example 4. If $\mu=(4,4,2,2)$ and $l=2$, then $\mu^{1 / l}=(4,2)$. There exists ten partitions of $\mu^{1 / l}:((4),(2)),((3,1),(2)),((2,2),(1,1)),((2,1,1),(1,1))$ etc. The partitions of the form $2 \underline{\kappa}$ for $\underline{\kappa} \vdash(4,2)$ are the following: $(8,4),(6,4,2),(4,4,4),(4,4,2,2)$, $(4,2,2,2,2),(8,2,2),(6,2,2,2),(2,2,2,2,2,2)$. Remark that it is possible for different $\underline{\kappa} \vdash \mu^{1 / l}$ that the resulting partitions $l \underline{\kappa}$ coincide, e.g., $2((2,2),(1,1))=$ $2((2,1,1),(2))=(4,4,2,2)$. If $\underline{\kappa}=((2,1,1),(2)) \vdash(4,2)$, then we have $m_{\underline{\kappa}}=$ $\left(\begin{array}{c}2 \\ 2,0\end{array}\right)\left(\begin{array}{c}2 \\ 1,1\end{array}\right)=2$. If $\underline{\kappa}^{\prime}=((2,2),(1,1)) \vdash(4,2)$, then $m_{\underline{\kappa}^{\prime}}=\left(\begin{array}{c}2 \\ 0,2\end{array}\right)\left(\begin{array}{c}2 \\ 2,0\end{array}\right)=1$.

The aim of this section is to prove the following theorem.

Theorem 5. Let l be a positive integer, and $\mu \vdash n$ an l-partition of a positive integer $n$. Then we have:

1. For a partition $\rho \vdash n$, the condition $Q_{\rho}^{\mu}\left(\zeta_{l}\right) \neq 0$ holds if and only if $\rho$ is a partition of the form $l \underline{\kappa}$ for some $\underline{\kappa} \vdash \mu^{1 / l}$.

2. For a partition $\rho=l \underline{\kappa}, \underline{\kappa} \vdash \mu^{1 / l}$, we have

$$
Q_{\rho}^{\mu}\left(\zeta_{l}\right)=\left(\sum_{\substack{\tau \vdash \mu^{1 / l} \\ \underline{\underline{\tau}}=\rho}} m_{\underline{\tau}} l^{l(\rho)}\right.
$$

where $l(\rho)$ denotes the length of $\rho$.

To prove the theorem, we first show a similar result for the Green polynomial $X_{\rho}^{\mu}(q)$ at $q=\zeta_{l}$, which is equivalent to Theorem 5 .

Proposition 6. Let $l$ be a positive integer, and $\mu \vdash n$ an $l$-partition. Then we have:

1. $X_{\rho}^{\mu}\left(\zeta_{l}\right) \neq 0 \Longleftrightarrow \rho=l \underline{\kappa}$ for some $\underline{\kappa} \vdash \mu^{1 / l}$.

2. For $\rho=l \underline{\kappa}, \underline{\kappa} \vdash \mu^{1 / l}$, we have

$$
X_{\rho}^{\mu}\left(\zeta_{l}\right)=(-1)^{n(l-1) / l}\left(\sum_{\substack{\frac{\tau}{l} \mu^{1 / l} \\ l \underline{\tau}=\rho}} m_{\underline{\tau}}\right) l^{l(\rho)} .
$$


Proof: Let $\mu \vdash n$ be an $l$-partition, and suppose that $\mu=\left(1^{m_{1}} 2^{m_{2}} \cdots n^{m_{n}}\right)$. Recall that

$$
X_{\rho}^{\mu}(q)=\left\langle Q_{\mu}^{\prime}(x ; q), p_{\rho}(x)\right\rangle .
$$

Let $q_{i}=m_{i} / l$ for each $i=1,2, \ldots, n$. By Proposition 2, we have

$$
Q_{\mu}^{\prime}\left(x ; \zeta_{l}\right)=\left\{Q_{\left(1^{l}\right)}^{\prime}\left(x ; \zeta_{l}\right)\right\}^{q_{1}}\left\{Q_{\left(2^{l}\right)}^{\prime}\left(x ; \zeta_{l}\right)\right\}^{q_{2}} \cdots\left\{Q_{((n / l)}^{\prime l)}\left(x ; \zeta_{l}\right)\right\}^{q_{n / l}} .
$$

Thus, we have

$$
X_{\rho}^{\mu}\left(\zeta_{l}\right)=\left\langle\prod_{i=1}^{n / l}\left\{Q_{\left(i^{l}\right)}^{\prime}\left(x ; \zeta_{l}\right)\right\}^{q_{i}}, p_{\rho}(x)\right\rangle .
$$

It follows from Proposition 3 and (3.2) that

$$
X_{\rho}^{\mu}\left(\zeta_{l}\right)=(-1)^{(l-1)\left(q_{1}+q_{2}+\cdots+q_{n / l}\right)}\left\langle\prod_{i=1}^{n / l}\left(p_{l} \circ h_{i}\right)^{q_{i}}(x), p_{\rho}(x)\right\rangle .
$$

Since

$$
h_{i}(x)=\sum_{\lambda \vdash i} z_{\lambda}^{-1} p_{\lambda}(x)
$$

we have

$$
p_{l} \circ h_{i}(x)=\sum_{\lambda \vdash i} z_{\lambda}^{-1} p_{l \lambda}(x) .
$$

It follows from (3.3) and (3.4) that

$$
X_{\rho}^{\mu}\left(\zeta_{l}\right)=(-1)^{(l-1) n / l} \sum_{\underline{\kappa} \vdash \mu^{1 / l}} \frac{z_{\rho}}{z_{\underline{\kappa}}}\left\langle p_{l \underline{\kappa}}(x), p_{\rho}(x)\right\rangle .
$$

Since $\left\{p_{\lambda}(x) \mid \lambda \in \operatorname{Par}\right\}$ is a orthogonal basis (2.2), it holds that

$$
X_{\rho}^{\mu}\left(\zeta_{l}\right) \neq 0 \Longrightarrow \rho=l \underline{\kappa}
$$

for some $\underline{\kappa} \vdash \mu^{1 / l}$.

Let $q_{i}=m_{i} / l$ for each $i$. Then the partitions $\underline{\kappa} \vdash \mu^{1 / l}$ are of the form

$$
\underline{\kappa}=\left(\kappa^{(11)}, \kappa^{(12)}, \ldots, \kappa^{\left(1 q_{1}\right)} ; \kappa^{(21)}, \kappa^{(22)}, \ldots, \kappa^{\left(2 q_{2}\right)} ; \cdots\right)
$$

where $\kappa^{(i j)}$ is a partition of $i$ for each $j=1,2, \ldots, q_{i}, i=1,2, \ldots, n / l$. Let $\rho$ be a partition of the form $\rho=l \underline{\kappa}$ for some $\underline{\kappa}=\left(\kappa^{(i j)}\right) \vdash \mu$. Let $\underline{\tau}=\left(\tau^{(i j)}\right)$ is a partition of $\mu^{1 / l}$ satisfying $l \underline{\tau}=\rho$. Suppose that

$$
\tau^{(i j)}=\left(1^{m_{1}^{(i j)}} 2^{m_{2}^{(i j)}} \cdots i^{m_{i}^{(i j)}}\right) \vdash i
$$


for each $i=1,2, \ldots, n / l$ and $j=1,2, \ldots, q_{i}$. If we set

$$
m_{k}=\sum_{(i j)} m_{k}^{(i j)}
$$

for each $k$, then we have

$$
\rho=\left(l^{m_{1}}(2 l)^{m_{2}} \cdots n^{m_{n / l}}\right) \vdash n .
$$

Therefore, by (3.5) and (3.6), we have

$$
X_{\rho}^{\mu}\left(\zeta_{l}\right)=(-1)^{(l-1) n / l} \sum_{\substack{\underline{\tau} \vdash \mu^{1 / l} \\ l \underline{\tau}=\rho}} \frac{z_{\rho}}{z_{\underline{\tau}}} .
$$

By (3.7), it holds for such a $\underline{\tau} \vdash \mu^{1 / l}$ that

$$
\begin{aligned}
\frac{z_{\rho}}{z_{\underline{\tau}}} & =\frac{l^{m_{1}} m_{1} !(2 l)^{m_{2}} m_{2} ! \cdots n^{m_{n / l}} m_{n / l} !}{\prod_{(i j)} 1^{m_{1}^{(i j)}} m_{1}^{(i j)} ! 2^{m_{2}^{(i j)}} m_{2}^{(i j)} ! \cdots i_{i}^{m_{i}^{(i j)}} m_{i}^{(i j)} !} \\
& =\frac{l^{m_{1}}}{\prod_{(i j)} 1^{m_{1}^{(i j)}}} \frac{(2 l)^{m_{2}}}{\prod_{(i j)} 2^{m_{2}^{(i j)}}} \cdots \times \frac{m_{1} !}{\prod_{(i j)} m_{1}^{(i j)} !} \frac{m_{2} !}{\prod_{(i j)} m_{2}^{(i j)} !} \cdots \\
& =l^{m_{1}+m_{2}+\cdots+m_{n / l}}\left(\begin{array}{c}
m_{1} \\
\left\{m_{1}^{(i j)}\right\}
\end{array}\right)\left(\begin{array}{c}
m_{2} \\
\left\{m_{2}^{(i j)}\right\}
\end{array}\right) \cdots\left(\begin{array}{c}
m_{n / l} \\
\left\{m_{n / l}^{(i j)}\right\}
\end{array}\right) \\
& =l^{l(\rho)} m_{\underline{\tau}},
\end{aligned}
$$

which proves the condition 2. Moreover, the condition 2 shows that $X_{\rho}^{\mu}\left(\zeta_{l}\right) \neq 0$ for $\rho=l \underline{\tau}, \underline{\tau} \vdash \mu^{1 / l}$, which completes the proof of the theorem.

To prove Theorem 5, we need the following auxiliary result.

Lemma 7. Let $l$ be a positive integer, and $\mu$ an $l$-partition. Then $(2 n(\mu)+(l-1) n) / l$ is an even integer.

Proof: Let us consider the case where $n / l$ is even. In this case, it is clear that $(l-1) n / l$ is even. It remains to show in this case that $2 n(\mu) / l$ is even. By the assumption, the Young diagram of the $l$-partition $\mu$ consists of even number of connected vertical $l$-strip. In the definition of $n(\mu)$, the sum of integers assigned to such a connected vertical $l$-strip is of the form $(l(l-1) / 2)+m l^{2}(m=0,1,2, \ldots)$. Hence $n(\mu)$ is a multiple of $l$, which shows that $2 n(\mu) / l$ is even.

Suppose that $n / l$ is odd. First we consider the case where $l$ is odd. In this case, it also holds that $n(\mu)$ is a multiple of $l$, and it is clear that $(l-1) n / l$ is even, since $l-1$ is even. Hence $(2 n(\mu)+(l-1) n) / l$ is even. Next we consider the case where $l$ is even. In this case, it is clear that $(l-1) n / l$ is odd. Hence we have to show that $2 n(\mu) / l$ is an odd integer. By the definition, $n(\mu)$ is the sum of $n / l$ positive integers of the form $(l(l-1) / 2)+m l^{2}\left(m \in \mathbf{Z}_{\geq 0}\right)$. Therefore, $2 n(\mu) / l$ is the sum of $n / l$ positive 
integers of the form $(l-1)+2 m l(m=0,1,2, \ldots)$. Since $n / l$ is odd, this is an odd integer.

We shall finish the proof of Theorem 5. By (2.1), we have

$$
Q_{\rho}^{\mu}\left(\zeta_{l}\right) \neq 0 \Longleftrightarrow X_{\rho}^{\mu}\left(\zeta_{l}\right) \neq 0
$$

By Proposition 6, this shows the first part of Theorem 5. Again by (2.1), it holds that

$$
Q_{\rho}^{\mu}\left(\zeta_{l}\right)=\zeta_{l}^{n(\mu)} X_{\rho}^{\mu}\left(\zeta_{l}^{-1}\right)
$$

By Proposition $6, X_{\rho}^{\mu}\left(\zeta_{l}^{-1}\right)$ does not depend on the particular choice of the primitive $l$ th root of unity. Hence, by (3.7) and Proposition 6, we have

$$
Q_{\rho}^{\mu}\left(\zeta_{l}\right)=(-1)^{\frac{2 n(\mu)+(l-1) n}{l}}\left(\sum_{\substack{\tau \vdash \mu^{1 / l} \\ l \underline{\tau}=\rho}} m_{\underline{\tau}} l^{l(\rho)}\right.
$$

Since $\mu$ is an $l$-partition, it holds from Lemma 7 that

$$
Q_{\rho}^{\mu}\left(\zeta_{l}\right)=\left(\sum_{\substack{\tau \\ l \underline{\tau}=\rho}} m_{\underline{\tau} / l}\right) l^{l(\rho)},
$$

which completes the proof of Theorem 5 .

Example 8. Let $\mu=(4,4,2,2)$ and $l=2$. Then $\mu$ is a 2-partition, and $\mu^{1 / 2}=(4,2)$. Let $\rho$ be a partition $(4,4,2,2)$. Then there exists two partition $((2,2),(1,1))$, $((2,1,1),(2))$ of $\mu^{1 / 2}=(4,2)$ satisfying $2 \underline{\kappa}=\mu^{1 / 2}$. For these $\underline{\kappa}$ 's, we have $m_{((2,2),(1,1))}=\left(\begin{array}{c}2 \\ 0,2\end{array}\right)\left(\begin{array}{c}2 \\ 2,0\end{array}\right)=1, m_{((2,1,1),(2))}=\left(\begin{array}{c}2 \\ 2,0\end{array}\right)\left(\begin{array}{c}2 \\ 1,1\end{array}\right)=2$. Therefore it holds that $Q_{\rho}^{\mu}\left(\zeta_{2}\right)=(1+2) 2^{4}=48$.

\section{Representation theory of the symmetric group}

In this section, we rephrase Theorem 5 in terms of representation theory of the symmetric group. It is known that the Green polynomial $Q_{\rho}^{\mu}(q)(\rho \vdash n)$ gives the graded character values of a certain graded representation $R_{\mu}$, called the DeConcini-ProcesiTanisaki algebra. The formula (3.1) shows that a certain combinatorial property of the algebra $R_{\mu}$, corresponding to an $l$-partition $\mu$, is interpreted in terms of representation theory of the symmetric group.

Let $n$ be a positive integer and $S_{n}$ the symmetric group of $n$ letters. If $\mu \vdash n$ be a partition, then there corresponds a homogeneous ideal $I_{\mu}$ of the polynomial ring $\mathbf{C}\left[x_{1}, x_{2}, \ldots, x_{n}\right]$, which is $S_{n}$-invariant. The symmetric group $S_{n}$ acts on the polynomial ring $\mathbf{C}\left[x_{1}, x_{2}, \ldots, x_{n}\right]$ as permutations of the variables, i.e., for $\sigma \in S_{n}$ Springer 
and $f=f\left(x_{1}, x_{2}, \ldots, x_{n}\right)$,

$$
(\sigma . f)\left(x_{1}, x_{2}, \ldots, x_{n}\right):=f\left(x_{\sigma(1)}, x_{\sigma(2)}, \ldots, x_{\sigma(n)}\right) .
$$

The DeConcini-Procesi-Tanisaki algebra $R_{\mu}$ is defined to be the quotient algebra

$$
R_{\mu}=\mathbf{C}\left[x_{1}, x_{2}, \ldots, x_{n}\right] / I_{\mu}
$$

of the polynomial ring. The algebra $R_{\mu}$ was first studied by C. de Concini and C. Procesi [3], as the $S_{n}$-module structure on the cohomology ring of a certain subvariety $X_{\mu}$ of the flag variety, the fixed point subvariety. T. Tanisaki [20] considers the generator of the defining ideal of $R_{\mu}$, and give a simple combinatorial description in terms of the partition $\mu$. For other topics related to a combinatorial point of view, see e.g., [4].

Since the defining ideal is homogeneous and $S_{n}$-invariant, the algebra $R_{\mu}$ has a structure of graded $S_{n}$-module

$$
R_{\mu}=\bigoplus_{d=0}^{n(\mu)} R_{\mu}^{d}
$$

i.e., each homogeneous component $R_{\mu}^{d}$ is $S_{n}$-submodule of $R_{\mu}$. The algebra $R_{\mu}$ is finite dimensional for each $\mu \vdash n$, and it is known that the dimension is given by the multinomial coefficient

$$
\operatorname{dim} R_{\mu}=\left(\begin{array}{c}
n \\
\mu_{1}, \mu_{2}, \ldots, \mu_{d}
\end{array}\right),
$$

if $\mu=\left(\mu_{1}, \mu_{2}, \ldots, \mu_{d}\right)$. With the Tanisaki generators, the structure of $R_{\mu}$ is easily seen for some special $\mu$. If $\mu=(n)$, then $R_{\mu}=\mathbf{C}$ the trivial representation of $S_{n}$. If $\mu=\left(1^{n}\right)$, then $R_{\mu}$ coincides with the coinvariant algebra $R_{n}$ of $S_{n}$, which is isomorphic to the left regular representation of $S_{n}$. For a general $\mu \vdash n$, it is known that, as an $S_{n}$-module,

$$
R_{\mu} \cong \operatorname{Ind}_{S_{\mu}}^{S_{n}} 1
$$

where 1 stands for the trivial representation of the Young subgroup $S_{\mu}$.

It is known that the Green polynomial $Q_{\rho}^{\mu}(q)$ gives the graded $S_{n}$-character of the algebra $R_{\mu}$. For each $d=0,1, \ldots, n(\mu)$, let char $R_{\mu}^{d}$ denote the character of the $S_{n}$-module $R_{\mu}^{d}$. Then the graded character $\operatorname{char}_{q} R_{\mu}$ of the graded $S_{n}$-module $R_{\mu}$ is defined by

$$
\operatorname{char}_{q} R_{\mu}(\rho)=\sum_{d=0}^{n(\mu)} q^{d} \operatorname{char} R_{\mu}^{d}(\rho)
$$


where $\rho$ is a partition of $n$, and $\operatorname{char} R_{\mu}^{d}(\rho)$ denotes the character value of the $S_{n}$-modules $R_{\mu}^{d}$ on the conjugacy class of cycle type $\rho$. Then we have (see, e.g., [4])

$$
\operatorname{char}_{q} R_{\mu}(\rho)=Q_{\rho}^{\mu}(q)
$$

for each $\rho \vdash n$.

In [12], it is verified that the Green polynomial $Q_{\rho}^{\mu}(q)$ has the following factorization formula.

Proposition 9. Let $\mu, \rho$ be partitions of a positive integer $n$, and $M_{\mu}$ the maximum value of the multiplicities $\left\{m_{1}(\mu), m_{2}(\mu), \ldots, m_{n}(\mu)\right\}$. Then there exists a polynomial $G_{\rho}^{\mu}(q)$ in $q$ with integer coefficients satisfying

$$
Q_{\rho}^{\mu}(q)=\frac{(1-q)\left(1-q^{2}\right) \cdots\left(1-q^{M_{\mu}}\right)}{(1-q)^{m_{1}(\rho)}\left(1-q^{2}\right)^{m_{2}(\rho)} \cdots\left(1-q^{n}\right)^{m_{n}(\rho)}} G_{\rho}^{\mu}(q)
$$

It is immediately follows from the formula that the Hilbert polynomial $H_{R_{\mu}}(q)=$ $\sum_{d} q^{d} \operatorname{dim} R_{\mu}^{d}$ of the algebra $R_{\mu}$ is of the form

$$
H_{R_{\mu}}(q)=\frac{(1-q)\left(1-q^{2}\right) \cdots\left(1-q^{M_{\mu}}\right)}{(1-q)^{n}} G_{\left(1^{n}\right)}^{\mu}(q),
$$

where $G_{\left(1^{n}\right)}^{\mu}(q)$ is a polynomial in $q$ with integer coefficients.

A proof of the following lemma, due to T. Oshima, is found in [14].

Lemma 10. Let $f(q)=a_{0}+a_{1} q+a_{2} q^{2}+\cdots$ be a polynomial in $q$ with integer coefficients. Let $l$ be a fixed positive integer such that $l \geq 2$, and for each $k=0,1, \ldots, l-1$ define

$$
c(k ; l):=\sum_{d \equiv k \bmod l} a_{d}
$$

Then these $c(k ; l)$ 's coincide with each other if and only if the polynomial $f(q)$ has roots of unity $\zeta_{l}^{j}$ as zeros for each $j=1,2, \ldots, l-1$.

We remark here that if the polynomial $f(q)=a_{0}+a_{1} q+a_{2} q^{2}+\cdots$ is of the form $\left(1+q+q^{2}+\cdots+q^{l-1}\right) g(q)$ for a certain polynomial $g(q)$, then it is clear by straight computation that the 'mod $l$-sums' $c(k ; l)$ of the coefficients of $f$ do not depend on $k$. Note that this is not clear for the case of the Hilbert polynomial $H_{R_{\mu}}(q)$.

Let $\mu$ be a partition, and $l$ a positive integer such that $2 \leq l \leq M_{\mu}$. (We exclude the case $l=1$, since it is trivial for our argument. ) For each $k=0,1, \ldots, l-1$, we define

$$
R_{\mu}(k ; l):=\bigoplus_{d \equiv k \bmod l} R_{\mu}^{d}
$$


Then it is immediately seen from Lemma 10 that these submodules $R_{\mu}(k ; l)(k=0$, $1, \ldots, l-1)$ have the same dimension. In the rest of this section, we shall consider the following problem that provides a representation theoretical interpretation for the property 'coincidence of dimension':

Find a subgroup $H(l)$ of $S_{n}$, and $H(l)$-modules $Z(k ; l)(k=0,1, \ldots, l-1)$ of equal dimension such that there exists a isomorphism of $S_{n}$-modules $R_{\mu}(k ; l) \cong$ $\operatorname{Ind}_{H(l)}^{S_{n}} Z(k ; l)$ for each $k=0,1, \ldots, l-1$.

Let $\mu$ be an $l$-partition. We shall define the product $a=a_{\mu}(l) \in S_{n}$ of cyclic permutations corresponding to $\mu$ and $l$. To avoid abuse of notation, we settle the definition through the following example. It is clear from the definition that the element $a$ corresponding to an $l$-partition has the order $l$.

Example 11 (The definition of $a$ ). Let $\mu$ be the partition $(3,3,2,2,2,2)$. Then the partition $\mu$ is a 2-partition. Consider the following standard Young tableau

$\begin{array}{ccc}1 & 2 & 3 \\ 4 & 5 & 6 \\ 7 & 8 & \\ 9 & 10 & \\ 11 & 12 & \\ 13 & 14 & .\end{array}$

Then the tableau decomposes modulo 2 into the following three parts:

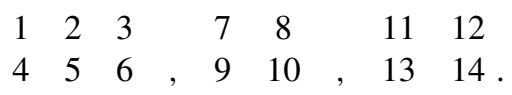

For each subtableau, we define the following products of cyclic permutations:

$$
\left(\begin{array}{llllll}
1 & 2 & 3 & 4 & 5 & 6 \\
4 & 5 & 6 & 1 & 2 & 3
\end{array}\right),\left(\begin{array}{cccc}
7 & 8 & 9 & 10 \\
9 & 10 & 7 & 8
\end{array}\right),\left(\begin{array}{cccc}
11 & 12 & 13 & 14 \\
13 & 14 & 11 & 12
\end{array}\right)
$$

Then $a$ is defined to be the product of these permutations:

$$
a=\left(\begin{array}{llllll}
1 & 2 & 3 & 4 & 5 & 6 \\
4 & 5 & 6 & 1 & 2 & 3
\end{array}\right)\left(\begin{array}{cccc}
7 & 8 & 9 & 10 \\
9 & 10 & 7 & 8
\end{array}\right)\left(\begin{array}{cccc}
11 & 12 & 13 & 14 \\
13 & 14 & 11 & 12
\end{array}\right)
$$

If we regard the partition $\mu=(2,2,2,2,2,2)$ as a 3-partition, then the corresponding element $a$ is defined to be the following product of cyclic permutations:

$$
a=\left(\begin{array}{llllll}
1 & 2 & 3 & 4 & 5 & 6 \\
3 & 4 & 5 & 6 & 1 & 2
\end{array}\right)\left(\begin{array}{cccccc}
7 & 8 & 9 & 10 & 11 & 12 \\
9 & 10 & 11 & 12 & 7 & 8
\end{array}\right)
$$


Define the subgroup $H_{\mu}(l)$ by

$$
H_{\mu}(l)=S_{\mu} \rtimes\left\langle a_{\mu}(l)\right\rangle \cong S_{\mu} \rtimes C_{l} .
$$

$H_{\mu}(l)$-modules $Z_{\mu}(k ; l)(k=0,1, \ldots, l-1)$ are defined to be the irreducible modules of $\langle a\rangle \cong C_{l}$, on which the Young subgroup $S_{\mu}$ acts trivially (hence all one dimensional).

Example 12. Let $\mu=(3,3,2,2)$ and $l=2$. Then $\mu^{1 / l}=(3,2)$ and $a=a_{\mu}(l)=$ $(1,4)(2,5)$

$(3,6)(7,9)(8,10)$. The subgroup $H_{\mu}(l)$ is defined to be the semi-direct product

$$
\left(S_{\{1,2,3\}} \times S_{\{4,5,6\}} \times S_{\{7,8\}} \times S_{\{9,10\}}\right) \rtimes\langle a\rangle \cong S_{\mu} \rtimes C_{2},
$$

where $S_{\{i, j, \ldots, k\}}$ denotes the symmetric group of the letters $\{i, j, \ldots, k\}$. The onedimensional $H_{\mu}(l)$-modules $Z_{\mu}(k ; l)(k=0,1)$ are by definition the irreducible $C_{2-}$ modules on which the Young subgroup $S_{\mu}$ acts trivially.

The aim of this section is to show the following theorem:

Theorem 13. Let l be a positive integer and $\mu$ an l-partition. With the notation above, we have

$$
R_{\mu}(k ; l) \cong{ }_{S_{n}} \operatorname{Ind}_{H_{\mu}(l)}^{S_{n}} Z_{\mu}(k ; l)
$$

for each $k=0,1, \ldots, l-1$.

We first show the equivalence of Theorem 13 and existence of a certain $S_{n} \times C_{l^{-}}$ isomorphism, which is originally suggested by T. Shoji for the case of coinvariant algebras. Let $l$ be a positive integer, and $\mu$ an $l$-partition. Consider the induced module $\operatorname{Ind}_{S_{\mu}}^{S_{n}} 1$, where 1 stands for the trivial representation of the Young subgroup $S_{\mu}$. As an $S_{n}$-module, this induced module is equivalent to $R_{\mu}$. We remark here that $R_{\mu}$ and $\operatorname{Ind}_{S_{\mu}}^{S_{n}} 1$ admit $S_{n} \times C_{l}$-module structures as follows. The $S_{n}$-module structures are natural ones. We define $C_{l}$-module structures in the sequel. Define the action of $C_{l}$ on $R_{\mu}$ by

$$
a^{j} \cdot x:=\zeta_{l}^{d j} x
$$

for $x \in R_{\mu}^{d}$. To define the $C_{l}$-module structure on $\operatorname{Ind}_{S_{\mu}}^{S_{n}} 1$, recall the following identification

$$
\operatorname{Ind}_{S_{\mu}}^{S_{n}} 1=\bigoplus_{\sigma \in S_{n} / S_{\mu}} \sigma \otimes \mathbf{C}
$$


where $\mathbf{C}$ denotes the trivial representation of $S_{\mu}$. Then we can define $C_{l}$-module structure on $\operatorname{Ind}_{S_{\mu}}^{S_{n}} 1$ by

$$
a^{j} .(\sigma \otimes 1):=\sigma a^{-j} \otimes 1,
$$

for each $\sigma \in S_{n} / S_{\mu}$ and $j$. It is clear from the definition that the $S_{n}$-action and the $C_{l}$-action commute with each other.

Proposition 14. Let $l \geq 2$ be a positive integer, and $\mu$ an l-partition. Then there exists $S_{n}$-isomorphisms

$$
R_{\mu}(k ; l) \cong S_{n} \operatorname{Ind}_{H}^{S_{n}} Z_{\mu}(k ; l), \quad k=0,1, \ldots, l-1
$$

if and only if the $S_{n} \times C_{l}$-modules $R_{\mu}$ and $\operatorname{Ind}_{S_{\mu}}^{S_{n}} 1$ are equivalent:

$$
R_{\mu} \cong S_{n} \times C_{l} \operatorname{Ind}_{S_{\mu}}^{S_{n}} 1
$$

Proof: Suppose that there exists an $S_{n} \times C_{l}$-isomorphism $R_{\mu} \cong \operatorname{Ind}_{S_{\mu}}^{S_{n}} 1$. If we remark that $\operatorname{Ind}_{S_{\mu}}^{S_{n}} 1=\bigoplus_{\sigma \in S_{n} / S_{\mu}} \sigma \otimes \mathbf{C}=\bigoplus_{\sigma \in S_{n} / S_{\mu} \rtimes C_{l}} \bigoplus_{j=1}^{l-1} \sigma a^{j} \otimes \mathbf{C}$, then it is easy to see that the eigenspace decompositions of both sides with respect to the action of $a$ give the isomorphisms $R_{\mu}(k ; l) \cong{ }_{S_{n}} \operatorname{Ind}_{H}^{S_{n}} Z(k ; l), k=0,1, \ldots, l-1$. The other direction of the proof is obtained by tracking back this argument.

The rest of this section is devoted to the proof of the isomorphism

$$
R_{\mu} \cong S_{n} \times C_{l} \operatorname{Ind}_{S_{\mu}}^{S_{n}} 1 .
$$

Since we are working on a field of characteristic zero, it is enough to show the character values of both sides coincide, i.e.,

$$
\operatorname{char} R_{\mu}\left(w, a^{j}\right)=\operatorname{char}_{\operatorname{Ind}_{S_{\mu}}^{S_{n}} 1\left(w, a^{j}\right)}
$$

for each $\left(w, a^{j}\right) \in S_{n} \times C_{l}$. Since the case $j=0$ is exactly the $S_{n}$-isomorphism $R_{\mu} \cong_{S_{n}} \operatorname{Ind}_{S_{\mu}}^{S_{n}} 1$, we may assume $j \geq 1$. Noticing that the action of the element $a$ on the homogeneous spaces $R_{\mu}^{d}$ is a scalar multiple, a slight consideration shows that the character value $\operatorname{char} R_{\mu}\left(w, a^{j}\right)$ coincides with the value of the Green polynomial $Q_{\lambda(w)}^{\mu}(q)$ at $q=\zeta_{l}^{j}$. Thus, we have to show

$$
Q_{\rho}^{\mu}\left(\zeta_{l}^{j}\right)=\operatorname{char} \operatorname{Ind}_{S_{\mu}}^{S_{n}} 1\left(w, a^{j}\right)
$$

for each $j=1,2, \ldots, l-1$. If we suppose that the $l$ th root of unity $\zeta_{l}^{j}$ is a primitive $m$ th root of unity, the order of the element $a^{j}$ coincides with $m$. Therefore, replacing $m$ with $l$ again, it is enough to show that

$$
Q_{\rho}^{\mu}\left(\zeta_{l}\right)=\operatorname{char} \operatorname{Ind}_{S_{\mu}}^{S_{n}} 1(w, a) .
$$


By Theorem 5, it suffices to show the following two conditions:

1. The condition char $\operatorname{Ind}_{S_{\mu}}^{S_{n}} 1(w, a) \neq 0$ holds if and only if the cycle type $\rho$ of $w$ is a partition of the form $l \underline{\kappa}$ for some $\underline{\kappa} \vdash \mu^{1 / l}$.

2. For an element $w \in S_{n}$ whose cycle type is of the form $\rho=l \underline{\kappa}, \underline{\kappa} \vdash \mu^{1 / l}$, we have

$$
\operatorname{char} \operatorname{Ind}_{S_{\mu}}^{S_{n}} 1(w, a)=\left(\sum_{\substack{\tau \vdash \mu^{1 / l} \\ l \underline{\tau}=\rho}} m_{\underline{\tau}} l^{l(\rho)},\right.
$$

where $l(\rho)$ denotes the length of $\rho$.

The 'only if' part of the first condition holds as follows. Recall the induced representation $\operatorname{Ind}_{S_{\mu}}^{S_{n}} 1$ has the realization $\operatorname{Ind}_{S_{\mu}}^{S_{n}}=\bigoplus_{\sigma \in S_{n} / S_{\mu}} \sigma \otimes \mathbf{C}$. Hence if char $\operatorname{Ind}_{S_{\mu}}^{S_{n}} 1(w, a) \neq 0$, then there should exist an element $\sigma \in S_{n} / S_{\mu}$ such that $\operatorname{char}(\sigma \otimes \mathbf{C})(w, a) \neq 0$. Since we have $(w, a)(\sigma \otimes 1)=w \sigma a^{-1} \otimes 1$, this forces that $w \sigma a^{-1} \equiv \sigma \bmod S_{\mu}$. Therefore, if char $\operatorname{Ind}_{S_{\mu}}^{S_{n}} 1(w, a) \neq 0$, then $w$ is conjugate to an element $\tau a \in H_{\mu}(l)$. Since cycle types of elements of $H_{\mu}(l)$ are of the form $l \underline{\kappa}$, $\underline{\kappa} \vdash \mu^{1 / l}$, then we have the 'only if' part of the condition 1 .

Suppose that an element $w$ satisfies the condition char $\operatorname{Ind}_{S_{\mu}}^{S_{n}} 1(w, a) \neq 0$. It follows from the assumption that $w$ is conjugate to an element of the subgroup $H_{\mu}(l)$. Since the argument depends only on the cycle type of $w$, we may assume that $w=\tau a \in H_{\mu}(l)$, where $\tau \in S_{\mu}$. Let the cycle type $\rho$ of $w$ be $\rho=l \underline{\kappa}$, where $\underline{\kappa} \vdash \mu^{1 / l}$. By the assumption, we have

$$
\operatorname{char}_{\operatorname{Ind}_{S_{\mu}}^{S_{n}} 1(w, a)=} \sum_{\substack{\sigma \in S_{n} / S_{\mu} \\ w \sigma a^{-1} \equiv \sigma \bmod S_{\mu}}} \operatorname{char}(\sigma \otimes \mathbf{C})(w, a)
$$

Consider $\sigma \in S_{n} / S_{\mu}$ such that $w \sigma a^{-1} \equiv \sigma$ modulo $S_{\mu}$. For such $\sigma$ 's, it is clear from the definition of the $S_{n} \times C_{l}$-module structure on $\sigma \otimes \mathbf{C}$ that $\operatorname{char}(\sigma \otimes \mathbf{C})(w, a)=1$. Therefore we have

$$
\operatorname{char} \operatorname{Ind}_{S_{\mu}}^{S_{n}} 1(w, a)=\sharp\left\{\sigma \in S_{n} / S_{\mu} \mid w \sigma a^{-1} \equiv \sigma \bmod S_{\mu}\right\} .
$$

It is possible to see that the number of representatives $\sigma \in S_{n} / S_{\mu}$ satisfying the condition $w \sigma a^{-1} \equiv \sigma \bmod S_{\mu}$ coincides with

$$
\left(\sum_{\substack{\frac{\pi}{l} \vdash \mu^{1 / l} \\ \underline{\pi}=\rho}} m_{\underline{\pi}} l^{l(\rho)}\right.
$$

which proves 2. (For details, see the following example.) Finally, it is immediately follows from the condition 2 that the 'if' part of the condition 1 holds. 
Example 15. Let $\mu=(3,3,2,2)$ and $l=2$. If char $\operatorname{Ind}_{S_{\mu}}^{S_{n}} 1(w, a) \neq 0$, then $w$ is conjugate to an element of $H_{\mu}(l)$, and the cycle type $\rho$ of $w$ is of the form $\rho=l_{\underline{\kappa}}$, $\underline{\kappa} \vdash \mu^{1 / l}=(3,2)$. Suppose that $w=(1,2) a=(1,4,2,5)(3,6)(7,9)(8,10)$. Then representatives $\sigma \in S_{n} / S_{\mu}$ satisfying $w \sigma a^{-1} \equiv \sigma$ modulo $S_{\mu}$ are for example $\sigma=$ $[1,2,3,4,5,6,7,8,9,10],[1,2,6,4,5,3,7,8,9,10]$, [1, 2, 7, 4, 5, 9, 3, 6, 9, 10], $[4,5,3,1,2,6,7,8,9,10]$ etc. On the other hand, the following type of representatives are also appropriate: $\sigma=[3,7,8,6,9,10,1,2,4,5],[6,7,8,3,9,10,1,2,4,5]$, $[3,7,8,6,9,10,4,5,1,2]$ etc. The number of representatives of the first type is $m_{((2,1,1),(1,1))} 2^{4}=\left(\begin{array}{c}4 \\ 2,2\end{array}\right)\left(\begin{array}{c}1 \\ 1,0\end{array}\right) 2^{4}$. That of the second type is $m_{((1,1,1,1),(2))} 2^{4}=\left(\begin{array}{c}4 \\ 4,0\end{array}\right)\left(\begin{array}{c}1 \\ 0,1\end{array}\right) 2^{4}$.

\section{Final Remark}

A problem of the type we consider in the previous section was first explicitly noticed by W. Kraśkiewicz and J. Weyman [7] for coinvariant algebras $R_{W}$ of Weyl groups $W$ of type $A, B, D$. They consider the problem for the case where $l$ is the Coxeter number, the order of Coxeter elements [6, p.74] of $W$. They show that each submodule $R_{W}(k ; l)$, similarly defined as in the previous section for $R_{\mu}$, is induced from the corresponding irreducible representation of the cyclic subgroup generated by a Coxeter element of $W$. As a consequence, we can see that these submodules $R_{W}(k ; l)$ are of equal dimension. In fact, T. A. Springer [18] had obtained implicitly these result for a wider setting. Let $W$ be a finite complex reflection group, $R_{W}$ the coinvariant algebra of $W$, and $l$ a regular number [18, Section 4] of $W$. Then it is possible to see from results in [18] that a similar statement holds and, as a consequence, those submodules $R_{W}(k ; l)$ are of equal dimension (see also [16]). The underlying subgroup is the cyclic subgroup generated by a regular element $[18$, Section 4] of order $l$. (Remark that the Coxeter number is a regular number.) Moreover, for a finite complex reflection group $W$, it is not difficult to see that if $l$ is a degree [6, p.59] of $W$, then the submodules $R_{W}(k ; l)$ are of equal dimension. (Remark that the regular numbers are degrees of $W$.)

Then, conversely, a new problem arises which asks a representation theoretical interpretation of the coincidence of dimensions. In [14], the authors consider this problem for the coinvariant of the symmetric group. The first answer to the problem was made for the coinvariant algebra for the symmetric group [14]. This result was generalized by C. Bonnafé, G. Lehrer, and J. Michel [1] for finite complex reflection groups. The problem considered in the present paper is another generalization of [14]. We make clear here the relation between the study of Green polynomials at roots of unity, which amounts to the study of Hall-Littlewood functions at roots of unity, and the problem for the algebra $R_{\mu}$ for special $\mu$ 's and special $l$ 's (see also [12]). In [13], we consider the problem for general $\mu$ 's and general possible $l$ 's. (Recently, the author was informed by T. Shoji that the problem considered in this article is given an affirmative answer in a largely generalized setting [17]. He considers the problem for the Springer representation of a connected reductive group over $\mathbf{C}$.)

\section{References}

1. C. Bonnafé, G. Lehrer, and J. Michel, “Twisted invariant theory for reflection groups,” preprint, 2005. 
2. J. Désarménien, B. Leclerc, and J.-Y. Thibon, "Hall-Littlewood Functions and Kostka-Foulkes Polynomials in Representation Theory," Seminaire Lotharingien de Combinatoire 32, 1994.

3. C. DeConcini and C. Procesi, "Symmetric functions, conjugacy classes, and the flag variety," Inv. Math. 64 (1981), 203-230.

4. A. M. Garsia and C. Procesi, "On certain graded $S_{n}$-modules and the $q$-Kostka polynomials," $A d v$. Math. 94 (1992), 82-138.

5. J. A. Green, "The character of the finite general linear groups," Trans. Amer. Math. Soc., 80 (1955), 402-447.

6. J. E. Humphreys, "Reflection Groups and Coxeter Groups," Cambridge studies in advances mathematics 29, Cambridge University Press, 1990.

7. W. Kraśkiewicz and J. Weyman, "Algebra of coinvariants and the action of Coxeter elements," Bayreuth. Math. Schr. 63 (2001), 265-284.

8. A. Lascoux, B. Leclerc, and Y. -Y. Thibon, "Fonctions de Hall-Littlewood et polynômes de KostkaFoulkes aux racines de l'unité,” C. R. Acad. Sci. Paris, 316, Ser. I (1993), 1-6.

9. A. Lascoux, B. Leclerc, and J. -Y. Thibon, "Green polynomials and Hall-Littlewood functions at roots of unity,” Euro. J. Comb. 15 (1994), 173-180.

10. G. Lusztig, "Green polynomials and singularities of unipotent classes," Adv. Math. 42 (1981), 169-178.

11. I. G. Macdonald, "Symmetric Functions and Hall Polynomials," 2nd ed., Oxford University Press, 1995.

12. H. Morita, "Decomposition of Green polynomial of type $A$ and DeConcini-Procesi-Tanisaki algebras of certain types," submitted.

13. H. Morita, "The Green polynomials at roots of unity and its application," submitted.

14. H. Morita and T. Nakajima, "The coinvariant algebra of the symmetric group as a direct sum of induced modules," Osaka J. Math. 42 (2005), 217-231.

15. A. O. Morris and N. Sultana, "Hall-Littlewood functions at roots of 1 and modular representations of the symmetric group," Math. Proc. Camb. Phil. Soc., 110 (1991), 443-453.

16. V. Reiner, D. Stanton, and P. Webb, "Springer's regular elements over arbitrary fields," preprint, 2004.

17. T. Shoji, "A variant of the induction theorem for Springer representations," preprint 2005.

18. T. A. Springer, "Regular elements of finite reflection groups," Invent. Math. 25 (1974), 159-198.

19. T. A. Springer, "Trigonometric sums, Green functions of finite groups and representations of Weyl groups," Inv. math. 36, (1976), 173-207.

20. T. Tanisaki, "Defining ideals of the closures of conjugacy classes and representations of the Weyl groups," Tohoku J. Math. 34 (1982), 575-585. 